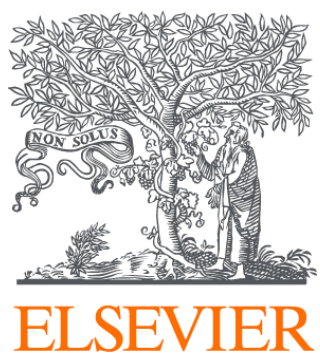

Since January 2020 Elsevier has created a COVID-19 resource centre with free information in English and Mandarin on the novel coronavirus COVID-

19. The COVID-19 resource centre is hosted on Elsevier Connect, the company's public news and information website.

Elsevier hereby grants permission to make all its COVID-19-related research that is available on the COVID-19 resource centre - including this research content - immediately available in PubMed Central and other publicly funded repositories, such as the WHO COVID database with rights for unrestricted research re-use and analyses in any form or by any means with acknowledgement of the original source. These permissions are granted for free by Elsevier for as long as the COVID-19 resource centre remains active. 


\section{Clinical Prevention and Population Health}

\section{Curriculum Framework for Health Professions}

Janet Allan, PhD, RN, Timi Agar Barwick, Suzanne Cashman, ScD, James F. Cawley, MPH, PA-C, Chris Day, MPH, Chester W. Douglass, DMD, PhD, Clyde H. Evans, PhD, David R. Garr, MD,

Rika Maeshiro, MD, MPH, Robert L. McCarthy, PhD, Susan M. Meyer, PhD, Richard Riegelman, MD, PhD,

Sarena D. Seifer, MD, Joan Stanley, PhD, RN, CRNP, Melinda Swenson, PhD, FNP,

Howard S. Teitelbaum, DO, PhD, MPH, Peggy Timothe, DDS, MPH, Kathryn E. Werner, MPA,

Douglas Wood, DO

Abstract: The Clinical Prevention and Population Health Curriculum Framework is the initial product of the Healthy People Curriculum Task Force convened by the Association of Teachers of Preventive Medicine and the Association of Academic Health Centers. The Task Force includes representatives of allopathic and osteopathic medicine, nursing and nurse practitioners, dentistry, pharmacy, and physician assistants. The Task Force aims to accomplish the Healthy People 2010 goal of increasing the prevention content of clinical health professional education. The Curriculum Framework provides a structure for organizing curriculum, monitoring curriculum, and communicating within and among professions. The Framework contains four components: evidence base for practice, clinical preventive services-health promotion, health systems and health policy, and community aspects of practice. The full Framework includes 19 domains. The title "Clinical Prevention and Population Health" has been carefully chosen to include both individual- and population-oriented prevention efforts. It is recommended that all participating clinical health professions use this title when referring to this area of curriculum. The Task Force recommends that each profession systematically determine whether appropriate items in the Curriculum Framework are included in its standardized examinations for licensure and certification and for program accreditation.

(Am J Prev Med 2004;27(5):471-476) @ 2004 American Journal of Preventive Medicine

\section{Introduction}

I ncreasingly, members of the health professions, policymakers, and the American public understand the importance of health promotion, disease prevention, and population health across a spectrum of issues affecting health, including chronic disease management, emerging infectious diseases, emergency preparedness, disparities in health and healthcare services, and the impact of behavior and lifestyle choices. ${ }^{1,2}$

From the American Association of Colleges of Nursing (Allan, Stanley), Association of Physician Assistant Programs (Barwick, Cawley), Community-Campus Partnerships for Health (Cashman, Seifer), Student Health Alliance (Day), American Dental Education Association (Douglass, Timothe), Association of Academic Health Centers (Evans), Association of Teachers of Preventive Medicine (Garr, Riegelman), Association of American Medical Colleges (Maeshiro), American Association of Colleges of Pharmacy (McCarthy, Meyer), National Organization of Nurse Practitioner Faculties (Swenson, Werner), and American Association of Colleges of Osteopathic Medicine (Teitelbaum, Wood)

Address correspondence and reprint requests to: Richard Riegelman MD, PhD, George Washington University School of Public Health and Health Services, 2300 Eye Street NW, Washington DC 20037. E-mail: sphrkr@gwumc.edu.

The full text of this article is available via AJPM Online at www.ajpm-online.net.
Increasingly, we realize the inadequacy of diseasebased, episodic, acute care intervention for addressing these issues. Nevertheless, a focus on prevention and population health continues to lag behind the emphasis on one-on-one treatment. Until prevention is thoroughly integrated into all aspects of our healthcare system, measurable progress addressing these issues will elude us.

An essential element of any effort to change a healthcare system must be the education of future clinicians who will practice new approaches in new contexts. Thus, an unambiguous emphasis on individual- as well as population-based prevention must be part of clinicians' education. ${ }^{3}$ Although a few innovative methods to integrate clinical prevention and population health into clinician training have been developed, ${ }^{4-8}$ no structured, comprehensive curriculum incorporates these topics into most health professionals' education.

The goal of implementing such a curriculum is not new. "Some 40 years of effort ... .to teach prevention as an integral part of clinical medicine...has met with limited success," wrote Barker and Jonas ${ }^{9}$ over 2 decades ago after reviewing the literature and their own 
experience. Despite past limited success, the time may be right for making real progress.

The events of fall 2001, the severe acute respiratory syndrome (SARS) epidemic, and West Nile virus have highlighted the critical role of prevention and public health. The Institute of Medicine (IOM) reports on medical errors and the quality of care highlighted the need to improve patient safety and restructure care systems. ${ }^{10,11}$ Another IOM report called for "transforming the content, methods, approaches, and settings used in health professions education" in response to the "changing needs of the population and changing demands of practice." ${ }^{12}$ Healthy People $2010^{13}$ encouraged the reexamination of clinical education by including an objective "to increase the proportion of schools of medicine, schools of nursing and health professional training schools whose basic curriculum for healthcare providers includes the core competencies in health promotion and disease prevention."

\section{Need for a New Curriculum}

Traditionally, each health profession designed, developed, and implemented its own curriculum framework. The approach presented here (1) assumes the need for and value of a common curriculum framework outlining the content that all health professions students should know and skills they all should have; (2) articulates how to organize such a framework; and (3) specifies what it should be called.

To integrate clinical prevention and population health into clinical practice, this approach assumes the need for effective interprofessional communications and collaboration. A mutually agreed upon curriculum framework articulating key elements of prevention and population health sciences provides not only the common, core subject matter, but also increases the opportunity for education and training in multiprofessional teams.

\section{Healthy People Curriculum Task Force Background}

The release of Healthy People 2010 joined the need for more effective prevention education with the need for greater interprofessional education and practice. Although not specifically recommended by Healthy People $2010,{ }^{13}$ if a prevention framework is to be widely used, it must be widely accepted across the core health professions-a goal that will be achievable if the framework is developed by leaders from the core clinical professions. The task of developing such a framework provided the impetus for the Association of Teachers of Preventive Medicine (ATPM) to join with the Associa- tion of Academic Health Centers to convene the Healthy People Curriculum Task Force, which is composed of a senior academic member and the executive director (or designee) from the following clinical health professional organizations:

Allopathic Medicine-Association of American Medical Colleges

Dentistry-American Dental Education Association

Nursing-American Association of Colleges of Nursing

Nurse Practitioners-National Organization of Nurse Practitioner Faculties

Osteopathic Medicine-American Association of Colleges of Osteopathic Medicine

Pharmacy-American Association of Colleges of Pharmacy

Physician Assistants-Association of Physician Assistant Programs

The Task Force also includes representation from the Student Health Alliance (a consortium of 11 health profession student organizations) and two resource groups, the Association of Schools of Public Health (ASPH) and CommunityCampus Partnerships for Health.

This unprecedented assemblage of diverse health professions stakeholders developed the Clinical Prevention and Population Health Curriculum Framework (Framework) that could be used by students from at least the seven represented health professions.

The progenitor for the Framework was conceived almost 20 years ago when ATPM assembled a group of leaders in prevention to articulate the basic prevention content for a comprehensive medical education curriculum. The resulting Inventory of Knowledge and Skills Relating to Disease Prevention and Health Promotion provided the guidepost for schools' efforts to broaden prevention training. Despite the intent that this document be "shared across many disciplines," it was associated with medical training and rarely used by other health professions. ${ }^{14-16}$

\section{Framework: Process and Intent}

Building on the above inventory, in 2003, the Task Force developed a preliminary curriculum framework in clinical prevention and population health. Widespread web-based review and evaluation of this document were sought from academics, students, practitioners, and through the participating organizations. Consultants recommended by ASPH provided comment on the preliminary document. In March 2004, representatives from all seven clinical professions on the Task Force unanimously approved the Framework.

The Framework provides a set of components and domains that constitute a foundation for education in clinical prevention and population health. The aim is 
to encourage each participating clinical health profession to review its curriculum recommendations and/or requirements and consider changes compatible with the Framework.

The Framework allows considerable flexibility for each clinical health profession to determine the depth of curriculum that is recommended, the timing for teaching the material, and the method(s) for delivery. The goal is to provide general recommendations and identify content areas that may require greater emphasis. It is also the intent to point out opportunities for interprofessional education and collaboration. The Framework is designed for degree programs rather than postgraduate or residency training, although it is hoped that these programs will build upon the Framework.

The Framework should be viewed as providing the foundation for a curriculum that spans the years of clinical health professional training. The curriculum content will generally need to be incorporated in more than one module or course in a degree program. Therefore, a mechanism for integrating this curricular content is important. Integration provides the opportunity to stress the interactive or ecologic nature of the factors affecting health and the development and outcome of disease (as stressed in recent IOM reports). ${ }^{17,18}$ The Framework also reflects the IOM's emphasis on health policy, ethics, and global health as components of public health education.

The name "clinical prevention and population health" has been carefully chosen to include both individual- and population-oriented preventive efforts as well as the interactions between them. It is recommended that participating health professions use this title when referring to this area of the curriculum.

\section{Framework: Content}

The Framework consists of four components-evidence base of practice, clinical preventive serviceshealth promotion, health systems and health policy, and community aspects of practice-with 19 domains. These components are recommended as a structure for organizing and monitoring curriculum, and communicating within and among disciplines. Within each component, the numbered domains are designed to outline content to reflect both individual clinical prevention and population health. The numbered domains allow each profession to identify the content considered relevant to its educational efforts. Finally, the listed items in each domain represent examples of the types of materials a particular profession may choose to encourage or require in its curriculum.

\section{Evidence Base of Practice}

\section{Epidemiology and biostatistics}

Rates of disease (e.g., incidence, prevalence, case fatality) Types of data (e.g., nominal, continuous, qualitative)
Statistical concepts (e.g., estimation [relative risk/ odds ratio and number needed to treat], statistical significance/confidence intervals, adjustment for confounding variables, causation)

2. Methods for evaluating health research literature

Study designs (e.g., surveys, observational studies, randomized clinical trials)

Quality measures (e.g., validity, accuracy, reproducibility, biases)

Sampling and statistical power

3. Outcome measurement, including quality and costs

Measures of mortality (e.g., infant mortality rates, life expectancy)

Measures that include quality of life/utility (e.g., quality-adjusted life years)

Measures that include cost (e.g., cost-effectiveness, incremental cost-effectiveness)

Measures of quality of health care (e.g., health status disparities, health plan employer data and information set [HEDIS])

4. Health surveillance

Vital statistics/legal documents (e.g., birth certificates, death certificates)

Disease surveillance (e.g., passive surveillance [reportable disease], active surveillance for epidemics and bioterrorism)

Biological, social, economic, geographic, and behavioral risk factors

5. Determinants of health

Burden of illness (e.g., distribution of morbidity and mortality by age, gender, race, socioeconomic status, geography)

Contributors to morbidity and mortality (e.g., genetic, behavioral, socioeconomic, environmental, health care [access and quality])

\section{Clinical Preventive Services-Health Promotion}

1. Screening

Approaches to testing and screening (e.g., range of normal, sensitivity, specificity, predictive value, target population)

Criteria for successful screening (e.g., effectiveness, benefits and harms, cost, patient acceptance)

Evidence-based recommendations

2. Counseling

Approaches to culturally appropriate behavioral change (e.g., counseling skill training, motivation)

Clinician-patient communication (e.g., patient participation in decision making, informed consent, risk communication, advocacy)

Criteria for successful counseling (e.g., effectiveness, benefits and harms, cost, patient acceptance)

Evidence-based recommendations

3. Immunization 
Approaches to vaccination (e.g., live vs dead vaccine, pre- vs post-exposure, boosters, target population, population-based immunity)

Criteria for successful immunization (e.g., effectiveness, benefits and harms, cost, patient acceptance)

Evidence-based recommendations

4. Chemoprevention

Approaches to chemoprevention (e.g., pre- vs postexposure, time limited vs long term)

Criteria for successful chemoprevention (e.g., effectiveness, benefits and harms, cost, patient acceptance)

Evidence-based recommendations

\section{Health Systems and Health Policy}

1. Organization of clinical and public health systems

Clinical health services (e.g., continuum of carehospital, ambulatory, home, long-term care)

Public health responsibilities (e.g., public health functions [Institute of Medicine]; ten essential services of public health)

Relationships between clinical practice and public health

2. Health services financing

Clinical services coverage and reimbursement (e.g., Medicare, Medicaid, employment based, uninsured)

Methods of financing of healthcare institution (e.g., hospitals, long-term care, community health centers)

Methods of financing of public health services

Other models (e.g., international comparisons)

3. Health workforce

Methods of regulation of professions and health care (e.g., certification, licensure, institutional accreditation)

Discipline-specific history, philosophy, roles, responsibilities

Racial/ethnic workforce composition including under-represented minorities

Relations of discipline to other healthcare professionals

Legal and ethical responsibilities of healthcare professionals (e.g., malpractice, healthcare information privacy, confidentiality)

4. Health policy process

Process of health policymaking (e.g., local, state, federal governments)

Methods for participation in the policy process (e.g., advocacy, advisory processes)

Impact of policies on health care and health outcomes including impacts on vulnerable populations

\section{Community Aspects of Practice}

1. Communicating and sharing health information with the public
Methods of assessing community needs/strengths and options for intervention (e.g., communityoriented primary care)

Media communications (e.g., strategies of using mass media, risk communication)

Evaluation of health information (e.g., websites, mass media, patient information [including literacy level and cultural sensitivity])

2. Environmental health

Sources, media, and routes of exposure to environmental contaminants (e.g., air, water, food)

Environmental health risk assessment and risk management (e.g., genetic, prenatal)

Environmental disease prevention focusing on susceptible populations

3. Occupational health

Risks from employment-based exposures

Methods for control of occupational exposures

Exposure and prevention in healthcare settings

4. Global health issues

Roles of international organizations

Disease and population patterns in other countries (e.g., burden of disease, population growth, health and development)

Effects of globalization on health (e.g., emerging and reemerging diseases/conditions)

5. Cultural dimensions of practice

Cultural influences on clinicians' delivery of health services

Cultural influences on individuals and communities (e.g., health status, health services, health beliefs)

Culturally competent health care

6. Community services

Methods of facilitating access to and partnerships for health care

Evidence-based recommendations for community preventive services

Public health preparedness (e.g., terrorism, natural disasters, injury prevention)

\section{Framework: Interpretation}

The following discussion addresses the four components. A full discussion of all elements of the Framework, including the 19 domains and general recommendations for implementation is included at www.atpm.org. This full document includes recommendations for timing and coordination of the curriculum, integration of curriculum content, and competency assessment.

\section{Evidence Base of Practice}

This component aims to operationalize the background needed to incorporate evidence into practice. The level of quantitative and qualitative analysis and study design understanding needed may vary from one 
profession to another. However, the numbered domains are designed to identify core competencies that are considered generally applicable for clinical health professional education. This component may be implemented using a variety of educational methods. Practice with structured reading of the health research literature will help ensure that students understand the relevance of these concepts.

\section{Clinical Preventive Services-Health Promotion}

This component aims to ensure that students not simply memorize recommended interventions, but understand the science needed to produce and interpret evidence-based recommendations for an intervention or service. This component is intended to parallel the structure established by the U.S. Preventive Services Task Force. ${ }^{19}$ The four domains within this component are intentionally structured in parallel, implying a similarity in approach and depth of understanding.

Each of the four domains could be subsumed into broader clinically oriented education. While it is desirable to connect each domain of this component with other clinically oriented education, it is important that a coherent approach be preserved for teaching the principles of screening, counseling, immunization, and chemoprevention.

Some clinical health professions may encourage or require knowledge of the content of specific evidencebased recommendations. However, that knowledge is considered separate from accomplishing the aims of this component.

\section{Health Systems and Health Policy}

A systematic approach to this component has not been part of most clinical health professional curricula. The development of a coherent curriculum that provides a framework for students to use as they experience the U.S. healthcare system is essential to accomplishing the intent of this component.

This component may be particularly amenable to interprofessional educational efforts, since the required level of knowledge is not likely to vary by discipline. Although ethical responsibilities are included under the Health Workforce domain, the integration of ethical issues throughout the entire framework is recommended.

Health policy is not generally required by current accreditation standards or included in most clinical health professions education curricula. Nevertheless, the Task Force overwhelmingly endorsed its inclusion here. The intent is to provide students with a basic understanding of policies that affect their practice and the health of their patients and communities, processes through which policies are developed, and opportunities to participate in policymaking.

\section{Community Aspects of Practice}

This component aims to integrate individual clinical prevention with the population health focus of the Framework. Community is defined broadly to include geographically defined communities as well as those defined by similar demographics, interests, or experiences (e.g., age, employment, diseases, health risks).

The need for community-based learning experiences outside traditional institutional settings is fundamental to this component. ${ }^{20}$ Service learning, communitybased clinical rotations (e.g., public health departments and community health centers), communitybased research, and international health experiences are possible ways to attain this goal. These might include education in the evaluation of web-based information, particularly from sites used by patients; learning firsthand about environmental and occupational exposures; and understanding the structure of international efforts to address current and emerging health problems. The CDC Guide to Community Preventive Services $^{21}$ can help support the development of prevention education across the health professions.

\section{Integration and Competency Assessment}

Integration of the four components is highly desirable. To integrate the curriculum, one might illustrate the options for intervention from primary prevention through rehabilitation; the level of intervention from the individual to the high-risk group to the general population; or the methods of behavioral intervention, including education, motivation, and training in counseling skills. Such integration may require incorporating specific curricular content near the end of the degree program.

Each clinical profession should address the methods used to evaluate students and to ensure their levels of competency. The Task Force recommends that each profession systematically determine whether appropriate items in the Framework are included as part of its standardized examinations for licensure and certification.

The Task Force encourages clinical health professions to explore creative methods for implementing the Framework using the opportunities it provides for interprofessional education. In addition, teaching the content contained in the Framework needs to emphasize the involvement of a range of health professionals beyond those represented on the Task Force.

\section{Future Task Force Activities}

The Framework presented here will be used through 2008-2009, when it will be extensively reviewed and revised. Data collection on inclusion of Framework content elements in the curricula of each of the seven professions is underway, in order to meet the require- 
ments of the Healthy People 2010 mid-course review in 2005. The Framework has been endorsed by several professions on the Task Force, and explorations with their accrediting bodies have begun.

To help disseminate and implement the Framework, the Task Force plans to develop an online Clinical Prevention and Population Health Resource Center. A web-based searchable database could eventually provide access to curricula for each of the 19 domains of the Framework, and searches would be possible to identify curricula for specific clinical disciplines and for teaching using a variety of formats. Linkages to educational consultants and continuing education programs may also be available through the Resource Center.

The Task Force will examine other options for implementing the Framework. For instance, the Task Force will examine the implications of the IOM recommendation that "all undergraduates should have access to education in public health." 17 To facilitate implementation of the Framework, graduate-level health professional programs might recommend an undergraduate public health course as part of their preprofessional preparation.

The Task Force succeeded in bringing together a wide spectrum of clinical health profession groups and developed a common framework for organizing, implementing, and monitoring curricula in clinical prevention and population health. Continued success will require building on this interprofessional communication and collaboration to develop models for interprofessional education.

This publication/project was made possible through Cooperative Agreements between the Office of Disease Prevention and Health Promotion (ODPHP) and the ATPM (award HPU010003-03), and the Health Resources and Services Administration (HRSA) and ATPM (award U78 HP 00010-05). Its contents are the responsibility of the authors and do not necessarily reflect the official views of ODPHP, HRSA, or ATPM.

\section{References}

1. McGinnis JM, Foege WH. Actual causes of death in the United States. JAMA 1993;270:2207-12.
2. McGinnis JM, Foege WH. Mortality and morbidity attributable to use of addictive substances in the United States. Proc Assoc Am Physicians 1999;111:109-18.

3. O'Neil EH. Pew Health Professions Commission. Recreating health professional practice for a new century. San Francisco: University of CaliforniaSan Francisco, Center for the Health Professions, 1998.

4. Epling JW, Sutphen SM, Morrow CB, Dismuke SE, Novick LF. A case-based curriculum for teaching clinical and population-based preventive medicine. Am J Prev Med 2003;24(suppl 4):83-169.

5. Hager M. Education for more synergistic practice of medicine and public health. New York: Josiah H. Macy, Jr. Foundation, 1999.

6. Drusin LM, Goss MEW, Horowitz SV, Reader GG. The clerkship in public health: a positive experience. Am J Prev Med 1992;8:58-61.

7. Novick LF, Greene C, Vogt RL. Teaching medical students about epidemiology: Utilizing a State Health Department. Public Health Rep 1985;100:401-5.

8. Finkel ML, Fein O. Teaching about the changing U.S. health care system: an innovative clerkship. Acad Med 2004;79:179-82.

9. Barker WH, Jonas S. The teaching of preventive medicine in American medical schools, 1940-1980. Prev Med 1981;10:674-88.

10. Institute of Medicine Health Care Quality Initiative. Crossing the quality chasm: a new health system for the twenty-first century. Washington DC: National Academy Press, 2001.

11. Greiner AC, Knebel E. Health professional education: a bridge to quality. Washington DC: National Academy Press 2003.

12. Kohn LT. Academic health centers: leading change in the 21st century. Washington DC: National Academy Press, 2003.

13. U.S. Department of Health and Human Services. Healthy People 2010. McLean VA: International Medical Publishing, 2000.

14. Wallace RB, Wiese WH, Lawrence RS, Runyan JW, Tilson HH. Inventory of knowledge and skills relating to disease prevention and health promotion. Am J Prev Med 1990;6:51-6.

15. Garr DR, Lackland DT, Wilson DB. Prevention education and evaluation in U.S. medical schools. Acad Med 2000;75:S14-21.

16. Pomrehn PR, Davis MV, Chen DW, Barker W. Prevention for the 21st century: setting the context through undergraduate medical education. Acad Med 2000:75:S5-13.

17. Hernandez L. Who will keep the public healthy? Educating public health professionals for the 21st century. Washington DC: National Academy Press, 2003.

18. Cuff PA, Vanselow N. Improving medical education: enhancing the behavioral and social science content of medical school curricula. Washington DC: National Academy Press, 2004.

19. U.S. Preventive Services Task Force. Guide to clinical preventive services. 2 vols. Washington DC: Agency for Healthcare Research and Quality, 2002.

20. Connors KM, Cashman S, Seifer SD, Unverzagt M. Advancing the healthy people 2010 objectives through community-based education: a curriculum planning guide. San Francisco CA: Community-Campus Partnerships for Health, 2003.

21. Community Preventive Services Task Force. Guide to community preventive services. Available at: www.phppo.cdc.gov/cdcrecommends. Accessed February 7, 2004. 